\title{
A new heuristic for full trie minimization
}

\author{
Meng Zhang ${ }^{\mathrm{a}}$, Dequan Chen ${ }^{\mathrm{a}}$, Yi Zhang ${ }^{\mathrm{b}}$, Guohang Song ${ }^{\mathrm{a}, *}$ \\ a College of Computer Science and Technology, Jilin University, Changchun 130012 China \\ b College of Electronics and Computer Science, Jilin Jianzhu University, Changchun 130118 China
}

*Corresponding author, e-mail: songguohang1988@sina.com

Received 25 Feb 2018

Accepted 28 Feb 2019

\begin{abstract}
Trie is a data structure with many applications. High space usage is the major drawback of the trie. The order-containing trie optimizes the space usage of the trie by rearranging symbols of strings. We present a new heuristic for building order-containing tries with small space. The heuristic is based on the following observation. For a given string set $P$, by moving the symbols on a position to the first position of strings in $P$, the trie of the resulting pattern set may have fewer nodes than that of the trie of $P$. We present an algorithm to find positions that yield the smallest such trie. The algorithm runs in $O(\|P\|)$ time and uses $O(|P| \log |P|)$ bits space, where $\|P\|$ is the number of total symbols in $P$, and $|P|$ is the number of patterns in $P$. By using this method recursively in trie constructions, we can build a trie with fewer nodes than the trie of $P$. We conduct several experiments that show the new heuristic builds smaller tires than previous work.
\end{abstract}

KEYWORDS: strings matching, compression, data structure, algorithm

MSC2010: 68W05 68W32

\section{INTRODUCTION}

The trie ${ }^{1}$ data structure is used in many applications, including bioinformatics, pattern matching, and computer security. High space usage is the major drawback of the trie. Much research focused on reducing the space of tries. Patricia tree ${ }^{2}$, also called path compressed trie, compresses the trie by deleting the nodes with one fan-out and labelling edges with a string, the number of nodes in the patricia tree of a string set $P=\left\{p_{1}, p_{2}, \ldots, p_{k}\right\}$ over an alphabet $\Sigma$ is not greater than $2|P|+1$. AhoCorasick automaton ${ }^{3}$ augments the trie with failure links. The multi-pattern matching can be solved in linear time and space by AC autotomata. Recently, Belazzougui et $\mathrm{al}^{4}$ used succinct data structures to implement AC automata with small space without slowing down.

De Maine and Rotwitt ${ }^{5}$ found that the order in which the characters are tested in building the trie has influences on the size of resulting tries. Using this idea, Comer and Sethi ${ }^{6}$ defined the ordercontaining trie (O-Trie). In an O-Trie the characters are tested in different orders along different paths from the root to leaves. The O-Trie construction reads the patterns in a specific order other than in serial. The orders of testing the characters are contained in the trie nodes. Using alternative orders, the resulting O-Tries have some interesting features, such as with fewer number of nodes. Comer and Sethi ${ }^{6}$ proved that to generate an O-Trie with the minimal number of nodes is NP-complete. Comer ${ }^{7,8}$ presented heuristic methods to build small O-Tries and gave thorough analysis. These methods may produce a trie larger than the trie for the original pattern set.

We present a new heuristic for the minimal full trie problem. Our approach guarantees that the number of nodes of the resulting O-Trie is not greater than that of the trie. Our method has the same time complexity of the greedy method of Comer ${ }^{7}$. It is based on a solution of the following problem. Assume that the patterns in $P$ are of the same length. By moving the symbol on the position $i$ of each pattern to the first position, we have a new set of patterns, say $P^{(i)}$. The problem is to search for a position $i$ such that the trie of $P^{(i)}$ has the smallest number of nodes. We present an algorithm that solves the problem in $O(\|P\|)$ time and $O(|P| \log |P|)$ bits space, where $\|P\|$ is the sum of lengths of all the patterns in $P$, and $|P|$ is the number of patterns in $P$. By applying the algorithm recursively to the trie construction, we can generate O-Tries such that the number of nodes is not greater that of the tries. 

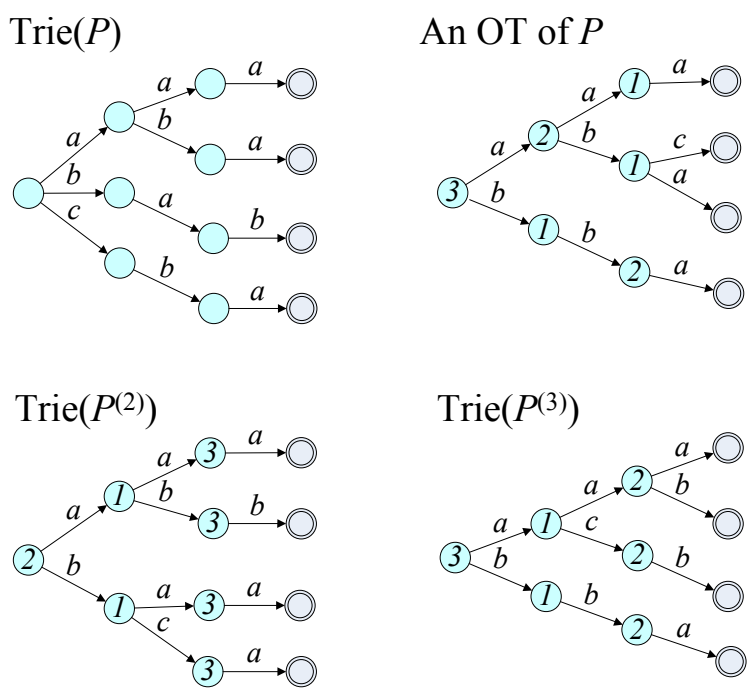

Fig. 1 Examples of an O-Trie and tries of $P=$ $\{a a a, a b a, b a b, c b a\}$, where terminal nodes are double circled. The number in a node $q$ is $\operatorname{Pos}(q)$.

\section{PRELIMINARIES}

Let $\Sigma$ denote the alphabet of $|\Sigma|$ symbols and $\varepsilon$ the empty string. Let $x=x[1] x[2] \cdots x[l]$ be a string over $\Sigma$ of length $l$. Given strings $x$ and $y$, we say that $x$ is a prefix of $x y$. Let $\operatorname{Pref}(P)$ denote the set of all prefixes of patterns in $P$. Define the trie of a pattern set as follows.

Definition 1 The trie of a string set $P$ is a tree with edges labelled by a symbol in $\Sigma$, the set of nodes is $V=\operatorname{Pref}(P)$, the set of edges is $E=\{(x, a, x a) \mid$ $x, x a \in \operatorname{Pref}(P), a \in \Sigma\}$. We use $\operatorname{Trie}(P)$ to denote the trie of $P$.

The node-depth of a trie node $u$, denoted by $\operatorname{depth}(u)$, is the number of nodes on the path from the root to $u$. We assume that all strings in the dictionary are of the same length $L$. The trie nodes are classified into levels according to their depths, the root is in level 1, and children of a node in level $l$ are in level $l+1$. The nodes corresponding to patterns in $P$ are called terminal nodes. The subtree rooted at the node pointed by an $a$-edge from the root of a tree is called an $a$-subtree of the tree. Throughout the paper we use $\mathscr{T}$ to denote $\operatorname{Trie}(P)$. We denote by $\operatorname{Size}(\mathscr{T})$ the number of nodes in $\mathscr{T}$. An example of the trie is given in Fig. 1, the upper left tree.

\section{ORDER-CONTAINING-TRIE}

Definition 2 A fractional string is a string with some positions that have no symbol, called blank positions.

We use symbol $\square$ to represent a blank position, when $\square$ is not in $\Sigma$. A fractional string is thus a string over $\Sigma \cup\{\square\}$. Let $p$ be a fractional string. If $u$ and $p$ are of the same size and for each position $i$ of $p$, $u[i]=p[i]$ or $u[i]=\square$, we call $u$ a fractional factor of $p$, or $u$ matches $p$. Function facins $(u, i, c)$ sets the blank position $i$ of $u$ to $c \in \Sigma$ and returns the resulting fractional string. For examples, $a \square a$ and $a a \square$ are fractional strings, $a \square a$ matches $a a a$, and facIns $(a \square a, 1, b)=a b a$.

We introduce the O-Trie using the notion of the fractional string. The set of nodes of an O-Trie, say $V$, is a subset of fractional factors of patterns in $P$, the root $q_{0}$ is $\square^{L}$, a string with $\square$ repeats for $L$ times. Each O-Trie involves a unique function Pos that is defined on each non-terminal node $q$ of the O-Trie and returns a blank position of $q$; if there is no blank position in $q$, $\operatorname{Pos}(q)$ is undefined. For $q \in V$, we denote the set of patterns in $P$ that match $q$ by $\operatorname{PatSet}(q)$. The set of symbols on position $\operatorname{Pos}(q)$ in each PatSet $(q)$ is denoted by $A(q)$. The set of edges starting from $q$ is defined as

$$
E(q)=\{(q, a, \operatorname{facIns}(q, \operatorname{Pos}(q), a)) \mid a \in A(q)\}
$$

The nodes corresponding to patterns in $P$ are terminal. An example of the O-Trie is given in Fig. 1, the upper right trie. Let $q$ be the node pointed by the $a$-edge from the root of this O-Trie. We have that $q=\square \square a$, PatSet $(q)=\{a a a, a b a, c b a\}$, and $A(q)=$ $\{a, b\}$, and $\operatorname{Pos}(q)=2$.

An O-Trie is a tree. If not, assume that there exists a node such that there are two paths from the root to this node. Let $i$ be the first position where the nodes are different in the two paths. According to the definition of O-Trie, the fractional string of the two nodes have one mismatch on a position. Thus descendants of the two nodes are all different, which contradicts the assumption.

For an edge $\left(q, a, q^{\prime}\right)$ in an O-Trie, node $q$ is the parent node of $q^{\prime}$, denoted by $q=\operatorname{parent}\left(q^{\prime}\right)$. A trie can be viewed as an O-Trie such that $\operatorname{Pos}(q)=$ $\operatorname{depth}(q)$. Comparing with a trie, O-Tries of the same pattern set may have different shapes and numbers of nodes. This difference is due to the Pos function of the O-Trie. 


\section{CONSTRUCTION OF O-TRIES}

We give a construction algorithm of an O-Trie. The implementation of function Pos will be given later when we introduce an instance.

The following is the framework of the breadthfirst construction algorithm of O-Tries, called BuildOT. The algorithm builds an O-Trie level by level. By definition, a node of an O-Trie corresponds to a fractional factor of some patterns in $P$. In generating level $i$ nodes from nodes in level $i-1$, each level $i-1$ node is extended by adding edges and children. We select a blank position of the $i-1$ level node and fill the bank position with a letter to form a new fractional factor that is a child. In the algorithm, we use PosSet(s) to denote the set of blank positions of node $s$ and $\operatorname{PatSet}(s)$ to denote the set of patterns that match $s$. Set $A$ is the set of symbols on position pos of each PatSet(s), and $P S[a]$ is the set of patterns in PatSet $(s)$ such that the symbol on the position pos is $a$.

Algorithm 1 Procedure BuildOT $(P, L)$

Step 1: Create a node $q_{0}$

Step 2: $\operatorname{PatSet}\left(q_{0}\right) \leftarrow P$

Step 3: $\operatorname{PosSet}\left(q_{0}\right) \leftarrow\{1, \ldots, L\}$

Step 4: $\operatorname{Expand}\left(q_{0}\right)$

Algorithm 2 Procedure Expand(s)

Step 1: if $\operatorname{PosSet}(s)=\varnothing$ then return

Step 2: $p o s \leftarrow \operatorname{Pos}(s)$

Step 3: set all elements of $P S$ to $\varnothing$, set $A$ to $\varnothing$

Step 4: for each $p \in \operatorname{PatSet}(s)$ do

1: $a \leftarrow p[p o s]$

2: if $a \neq \varepsilon$ then add $p$ to $P S[a]$ if $a \notin A$ then Add $a$ to $A$

Step 5: for each $a \in A$ do

1: create a node $s^{\prime}$

2: create an edge $\left(s, a, s^{\prime}\right)$

3: $\operatorname{PatSet}\left(s^{\prime}\right) \leftarrow P S[a]$

4: $\operatorname{PosSet}\left(s^{\prime}\right) \leftarrow \operatorname{PosSet}(s)-\{$ pos $\}$

5: $\operatorname{Expand}\left(s^{\prime}\right)$

\section{Construction of O-Tries with fewer nodes}

In this section, we present an instance of function Pos such that the corresponding O-Tries have fewer nodes than that of tries.

Let $p$ be a string of length $L$. For $1 \leqslant l \leqslant$ $L$, we construct a string $u$ such that $u[1]=p[l]$, $u[j]=p[j-1]$ for $2 \leqslant j<l$, and $u[j]=p[j]$ for $j>l$. We denote $u$ by $p^{(l)}$. For $P$, define $P^{(l)}=$ $\left\{p_{1}^{(l)}, p_{2}^{(l)}, \ldots, p_{k}^{(l)}\right\}$.

We define function $\operatorname{ML}(P)$ such that for a pattern set $P, \operatorname{ML}(P)$ returns a level $x$ of $P$ such that $\operatorname{Size}\left(\operatorname{Trie}\left(P^{(x)}\right)\right)$ is the minimum among Size $\left(\operatorname{Trie}\left(P^{(l)}\right)\right), 1 \leqslant l \leqslant L$.

We use ML to implement Pos in $\operatorname{BuildOT}(P)$ as follows. Given a node $s$, we can build a pattern set from PatSet $(s)$ and $\operatorname{PosSet}(s)$. Let $\operatorname{PatSet}(s)=$ $\left\{p s_{1}, \ldots, p s_{t}\right\}$, PosSet $(s)=\left\{l_{1}, \ldots, l_{c}\right\}$, and $p s_{i}^{\prime}=$ $p s_{i}\left[l_{1}\right] p s_{i}\left[l_{2}\right] \cdots p s_{i}\left[l_{c}\right]$. We define the pattern set $\operatorname{Pat}(s)=\left\{p s_{1}^{\prime}, \ldots, p s_{t}^{\prime}\right\}$. We implement $\operatorname{Pos}(s)$ by $\operatorname{ML}(\operatorname{Pat}(s))$. According to the definition of ML we have the following result.

Theorem 1 Let $P$ be a pattern set. Let $T$ be the trie generated by BuildOT(P) using $\mathrm{ML}(\operatorname{Pat}(\cdot))$ as $\operatorname{Pos}(\cdot)$. We have Size $(T) \leqslant \operatorname{Size}(\mathscr{T})$, where $\mathscr{T}$ denotes $\operatorname{Trie}(P)$.

When context is clear, we use $\mathscr{T}^{l}$ to denote $\operatorname{Trie}\left(P^{(l)}\right)$. The time and space complexities of computing $\operatorname{ML}(P)$ by building each $\mathscr{T}^{l}$ are $O\left(\|P\|^{2}\right)$ and $O(\|P\|)$. We present here a faster algorithm that only scans $P$ for one pass, which has time and space complexities $O(\|P\|)$ and $O(|P|)$, respectively. We first present a naive algorithm based on a relationship between $\mathscr{T}$ and $\mathscr{T}^{l}$, and then give a faster method.

\section{A naive algorithm to compute function ML}

Given $\mathscr{T}$, we construct the $a$-subtree of $\mathscr{T}^{l}, 1 \leqslant l \leqslant$ $L$, as follows.

Step 1: Mark all the $l$-level nodes that have an $a$ edge, and mark the subtrees rooted at the nodes pointed by these $a$-edges. Mark the paths from marked $l$-level nodes to the root.

Step 2: For each marked $l$-level node $s$, redirect the edge, which points to $s$, to the destination of the $a$-edge from $s$.

Step 3: Delete all marked $l$-level nodes and edges from them. Delete all un-marked nodes and edges.

Using this method, we generate the $a$-subtree of $\mathscr{T}^{l}$ for each symbol $a$ to have $\mathscr{T}^{l}$, and the function ML is then computed. We give an illustration in Fig. 2.

Next, we give a faster method base on the followed observation. The differences between $\mathscr{T}$ and $\mathscr{T}^{l}$ lie in the first $l$ levels, for the subgraphs of $\mathscr{T}$ and $\mathscr{T}^{l}$ from level $l+1$ to level $L$ are isomorphic. Thus the difference of the number of nodes of $\mathscr{T}$ and $\mathscr{T}^{l}$ equals the difference of the number of nodes 

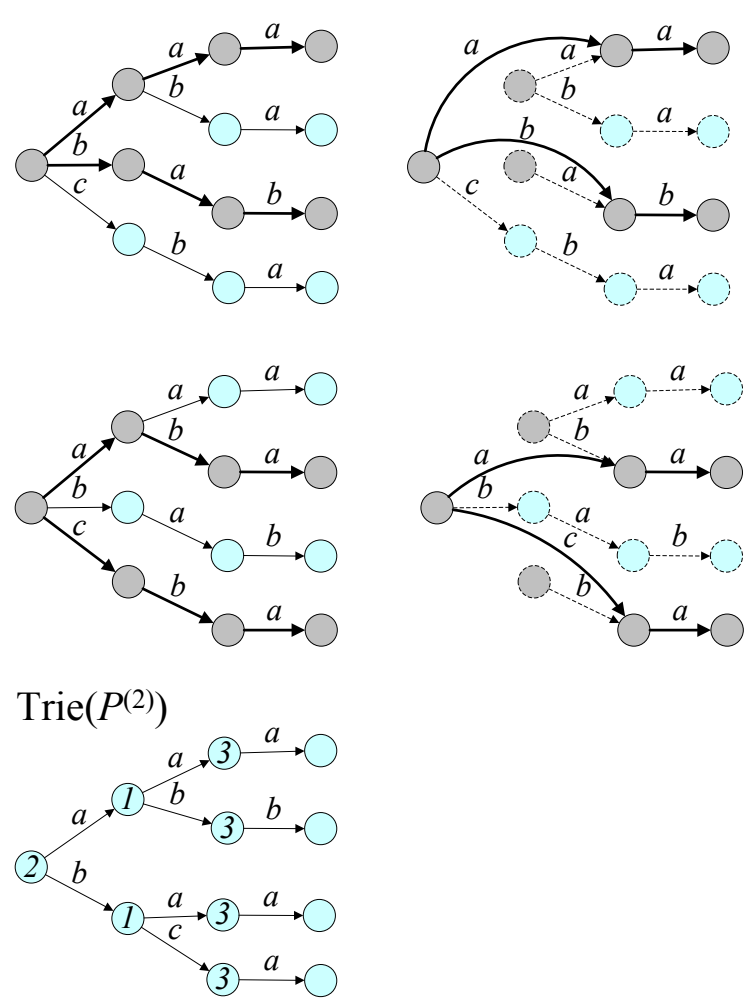

Fig. 2 The construction of $\operatorname{Trie}\left(P^{(2)}\right)$. Dark nodes and edges are marked, and dotted ones are deleted. The trees in the first line are marked Trie $(P)$ 's according to $a$ and $b$. The trees in the second line are $a$-subtree and $b$-subtree of $\operatorname{Trie}\left(P^{(2)}\right)$ generated from the marked tries. $\operatorname{Trie}\left(P^{(2)}\right)$ is built from the two trees.

in the first $l$ levels of $\mathscr{T}$ and $\mathscr{T}^{l}$. Define $\Delta(l)=$ $\operatorname{Size}\left(\mathscr{T}^{l}\right)-\operatorname{Size}(\mathscr{T})$. The minimal $\mathscr{T}^{l}$ corresponds to the minimal $\Delta(l)$. We count the number of nodes in the first $l$ levels of $\mathscr{T}^{l}$ efficiently from $\mathscr{T}$ without building $\mathscr{T}^{l}$ explicitly. Given $\mathscr{T}$, the counting procedure Count $\mathrm{T}(l, a)$ is as the following.

Algorithm 3 Procedure Count $(l, a)$

Step 1: Mark the root, count $\leftarrow 1$

Step 2: for each $l$-level node $s$ that has a-edge do while $s$ is not marked do

1: mark $s$ and the edge to $s$

2: count $\leftarrow$ count +1

3: $s \leftarrow \operatorname{parent}(s)$

Step 3: return count

CountT $(l, a)$ first marks the root of $\mathscr{T}$. For each $l$ level node $s$ that has an $a$-edge, it marks the path from $s$ to the root and counts the newly marked nodes. In the procedure, after the for loop, the tree of the marked nodes and edges is isomorphic to the first $l$ levels of the $a$-subtree of $\mathscr{T}^{l}$, and we know the number of nodes. We summarize the result as follows.

Proposition 1 Given the trie $\mathscr{T}$ of $P$, procedure Count $(l, a)$ returns the number of nodes of the first l levels of a-subtree of $\mathscr{T}^{l}$.

Proof: Each path from root to an $l$-level node with an $a$-edge spells out a prefix of length $l$, denote the set of such prefixes by $B$. Procedure $\operatorname{CountT}(l, a)$ marks the trie of $B$ and returns the number of nodes of the trie. By appending $a$ to the end of each string in $B$, we have the set of prefixes of $P$ of length $l+1$ that end with $a$. Hence the trie of $B$ is isomorphic to the first $l$ levels of the $a$-subtree of $\mathscr{T}^{l}$. Thus procedure Count $(l, a)$ returns the number of nodes of the first $l$ levels of the $a$-subtree of $\mathscr{T}^{l}$.

\section{Properties of trees used to implement ML}

Procedure Count T works when $\mathscr{T}$ is given. To count the nodes in $\mathscr{T}^{l}$, many nodes are accessed redundantly by Count $(l, a)$ for many $a$ 's. We introduce a faster and space efficient algorithm to count $\mathscr{T}^{l}$. The method does not need $\mathscr{T}$ or backtracking. It generates at most $|P|$ nodes in the running and the time complexity for compute $\operatorname{ML}(P)$ is $O(\|P\|)$.

We first give some necessary notions. Let $n_{1}$ and $n_{2}$ be nodes in the $l$ level of $\mathscr{T}$. We denote the least common ancestor (LCA) of $n_{1}$ and $n_{2}$ by $n=\operatorname{LCA}\left(n_{1}, n_{2}\right)$. The encounter distance between $n_{1}$ and $n_{2}$ is defined as $D\left(n_{1}, n_{2}\right)=l-\operatorname{depth}(n)$.

Definition 3 Define a partial order $<$ on the set of nodes of $\mathscr{T}$ as follows.

(i) If $n_{1}, \ldots, n_{k}$ are children of a node, then $n_{1}<$ $n_{2}<\cdots<n_{k}$.

(ii) For nodes $n, m$ in the same level, if parent $(n)<$ parent $(m)$, then $n<m$.

Let $n, m$ be nodes in the same level and $n<m$. If there does not exist node $n^{\prime}$ such that $n<n^{\prime}<m$, then $n$ is called the predecessor of $m$, denoted by $n=\operatorname{pre}(m)$.

The order in which the nodes are generated by BuildOT $(P)$ satisfies Definition 3. The computation of the number of nodes of $\mathscr{T}^{l}$ and that of encounter distance are based on the following results on trees.

Proposition 2 Let $n_{1}<n_{2}<n$ be nodes in the same level. We have $D\left(n, n_{2}\right) \leqslant D\left(n, n_{1}\right)$.

Proof: If $\operatorname{LCA}\left(n_{1}, n\right)=\operatorname{LCA}\left(n_{2}, n\right)=q$, then $D\left(n, n_{2}\right)=D\left(n, n_{1}\right)$. Let $\operatorname{LCA}\left(n_{1}, n\right) \neq \operatorname{LCA}\left(n_{2}, n\right)$. 
Then $\operatorname{LCA}\left(n_{1}, n_{2}\right)$ is either $\operatorname{LCA}\left(n_{1}, n\right)$ or $\operatorname{LCA}\left(n_{2}, n\right)$. Let $q_{1}=\operatorname{LCA}\left(n, n_{1}\right)$ and $q_{2}=\operatorname{LCA}\left(n, n_{2}\right)$. If $D\left(n, n_{2}\right)>D\left(n, n_{1}\right)$, then $q_{2}=\operatorname{LCA}\left(n_{1}, n_{2}\right)$. Let $q_{2}^{\prime}$ be the node in the path from $n_{2}$ to $m$ of the same depth of $q_{1}$. We have $q_{1}<q_{2}^{\prime}$, for $n_{1}<n_{2}$. As $q_{1}$ is an ancestor of $n$ and $q_{2}^{\prime}$ is an ancestor of $n_{2}$, we have $n<n_{2}$, which contradicts $n>n_{2}$. Hence $D\left(n, n_{2}\right) \leqslant D\left(n, n_{1}\right)$.

The number of nodes in the first $l$ levels of the $a$-subtree of $\mathscr{T}^{l}$ is computed by the encounter distances between adjacent nodes according to $<$ in the level $l$ of the subtree.

Proposition 3 Let $n_{1}<n_{2} \cdots<n_{k}$ be the set of $l$ level nodes that have an a-edge. The number of nodes in the first $l$ levels of a-subtree of $\mathscr{T}^{l}$ is $l+$ $\sum_{j=2}^{k} D\left(n_{j}, n_{j-1}\right)$.

Proof: In the running of CountT $(l, a)$, we denote the tree of the marked nodes after the $j$ th iteration by $T_{j}$. After backtracking $n_{1}$, we marked a path from $n_{1}$ to the root, say $T_{1}$, to have $\operatorname{Size}\left(T_{1}\right)=l$. After the $t$ th iteration of Count $(l, a)$, we assume that

$$
\begin{aligned}
\operatorname{Size}\left(T_{t}\right) & =l+\sum_{j=2}^{t} D\left(n_{j}, n_{j-1}\right) \\
& =\operatorname{Size}\left(T_{t-1}\right)+D\left(n_{t-1}, n_{t}\right) .
\end{aligned}
$$

In the $(t+1)$ th iteration of Count $(l, a)$, let $q$ be the first node in $T_{t}$ in the path from $n_{t+1}$ to the root. We have that $q$ is the LCA of $n_{t}$ and $n_{t+1}$, which is also the deepest among LCAs of $n_{j}$ and $n_{t+1}$. If it is not the case, then there are some nodes on the path that is a part of $T_{t}$, contradicting the definition of $q$. By Proposition 2, $D\left(n_{t+1}, n_{t}\right)$ is the minimum among $\left\{D\left(n_{t+1}, n_{j}\right) \mid 1 \leqslant j \leqslant t\right\}$. Hence the number of nodes in the path that is not $q$ is $D\left(n_{t+1}, n_{t}\right)$. Thus

$$
\begin{aligned}
\operatorname{Size}\left(T_{t+1}\right) & =\operatorname{Size}\left(T_{t}\right)+D\left(n_{t}, n_{t+1}\right) \\
& =l+\sum_{j=2}^{t+1} D\left(n_{j}, n_{j-1}\right) .
\end{aligned}
$$

By Proposition 1, the number of nodes of the first $l$ levels of $\mathscr{T}^{l}=l+\sum_{j=2}^{k} D\left(n_{j}, n_{j-1}\right)$.

The encounter distance between two nodes in the same level is computed as follows.

Proposition 4 Let $n_{1}<n_{2} \cdots<n_{c}$ be the set of nodes in the same level. For $i<j$, we have $D\left(n_{i}, n_{j}\right)=$ $\operatorname{Max}\left\{D\left(n_{t-1}, n_{t}\right) \mid i<t \leqslant j\right\}$.

Proof: For $j=i+1, D\left(n_{i}, n_{j}\right)=\operatorname{Max}\left\{D\left(n_{t-1}, n_{t}\right) \mid\right.$ $i<t \leqslant j\}$. Assume that for $j>i+1, D\left(n_{i}, n_{j}\right)=$
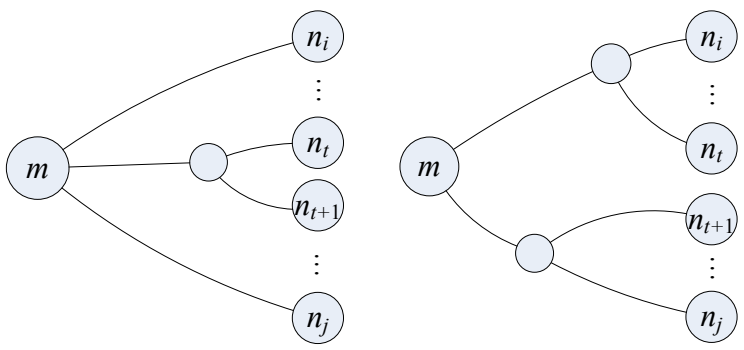

Fig. 3 There exist adjacent nodes $n_{t}$ and $n_{t+1}$, where $i \leqslant$ $t<j$ such that $\operatorname{LCA}\left(n_{t}, n_{t+1}\right)=\operatorname{LCA}\left(n_{i}, n_{j}\right)$.

$\operatorname{Max}\left\{D\left(n_{t-1}, n_{t}\right) \mid i<t \leqslant j\right\} . \quad$ If $D\left(n_{i}, n_{j}\right)=$ $D\left(n_{i}, n_{j+1}\right)$, then $D\left(n_{i}, n_{j+1}\right)=\operatorname{Max}\left\{D\left(n_{t-1}, n_{t}\right) \mid i<\right.$ $t \leqslant j+1\}$. Assume that $D\left(n_{i}, n_{j}\right)<D\left(n_{i}, n_{j+1}\right)$. Let $m=\operatorname{LCA}\left(n_{i}, n_{j+1}\right)$. We have $\operatorname{LCA}\left(n_{j}, n_{j+1}\right)=m$. Hence we prove the proposition.

An illustration of Proposition 4 is shown in Fig. 3.

Let $n_{1}<n_{2} \cdots<n_{c}$ be the set of $l$ level nodes. Define $D P^{l}$ as an array of $k$ entries such that $D P^{l}[i]=\operatorname{LCA}\left(n_{i}, n_{i-1}\right), n_{0}=n_{1}$. According to Proposition 4, the computation of $D\left(n_{i}, n_{j}\right)=l-$ $\operatorname{depth}\left(\operatorname{LCA}\left(n_{i}, n_{j}\right)\right), i>j$, can be reduced to the problem of range minimum query (RMQ) on $D P^{l}$. The problem of RMQ has been studied extensively. For a sequence of $n$ integers, the work ${ }^{9-12}$ solve the RMQ problem in $O(1)$ query time using $O(n)$ space and $O(n)$ preprocessing time. The array $D P^{l}$ can be computed from $D P^{l-1}$ using the following observation.

Proposition 5 Let $s, s^{\prime}$ be nodes such that $s=\operatorname{pre}\left(s^{\prime}\right)$. If $s$ and $s^{\prime}$ are siblings, we have $D\left(s, s^{\prime}\right)=1$, otherwise, $D\left(s, s^{\prime}\right)=D\left(\right.$ parent $(s)$, parent $\left.\left(s^{\prime}\right)\right)+1$.

\section{A faster algorithm to compute ML}

Based on Proposition 3-5, we design the algorithm Cholevel to compute $\Delta(1), \ldots, \Delta(L)$ in building $\mathscr{T}$ breadth-firstly. In contrast to procedure Count T, any node is accessed once without redundant visiting. In expanding the nodes in level $l$, the algorithm computes $\Delta(l)$. In the running, the algorithm only keeps the information of some nodes in level $l$ and $l+1$, the number of which does not exceed $|P|+1$.

In round $l$, the algorithm generates the nodes in level $l+1$ in the increasing order defined in Definition 3, and computes the array of the depth of the LCA node of each $(l+1)$-level node and its predecessor. We build the RMQ data structure for the array. Using the RMQ structure, we compute the encounter distance between nodes (Proposition 4) 
in $O(1)$ time and count the number of nodes of the first $l$ levels of the trie $\mathscr{T}^{l}$ by Proposition 3. we compute $\Delta(l)$ using the number of nodes of the first $l$ levels of the trie $\mathscr{T}$. By finding out the $l$ with the maximum $\Delta(l)$, we implement the function ML.

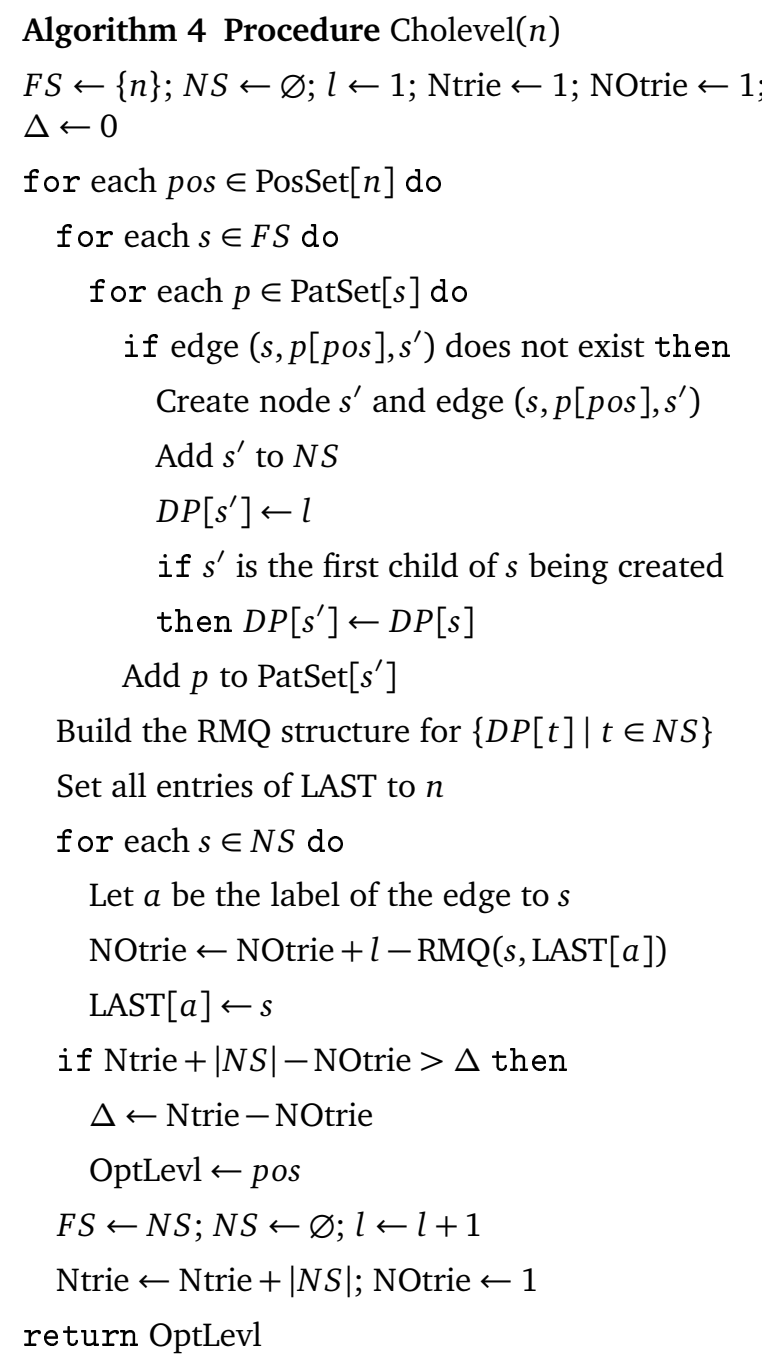

In the algorithm, $l$ is the number of levels processed, NOtrie is the number of nodes in the first $l$ levels of $\mathscr{T}^{l}$, Ntrie is that of $\mathscr{T}$, and pos is the position of the pattern set being used. The algorithm uses FS to keep all the nodes in level $l$ that are not expanded and $N S$ to keep the children of nodes in level $l$. For array $D P, D P[s]=\operatorname{depth}(\operatorname{LCA}(s, \operatorname{pre}(s)))$. For a node $s$ in level $l, D(s, \operatorname{pre}(s))=l-D P[s]$. According to Proposition 5, $D P[s]$ is computed from $D P[$ parent $(s)]$. The RMQ structure is built upon the array of $D P$ values of nodes in NS. Let $n_{1}<$ $n_{2}<\cdots<n_{c}$ be the set of nodes in NS. Function $\operatorname{RMQ}\left(n_{i}, n_{j}\right), i<j$, returns the minimum value in $D P\left[n_{i}\right], D P\left[n_{i+1}\right], \ldots, D P\left[n_{j}\right]$.
Let $n^{\prime}$ be the newly generated node and pointed by an edge labelled by $a$. Table LAST has $|\Sigma|$ entries for each character in $\Sigma$, where LAST $[a]$ is the node in $N S$, which is the latest node being created and pointed by an $a$-edge. By definition, the encounter distance between $n^{\prime}$ and LAST $[a]$ is $l$-depth(LCA $\left.\left(n^{\prime}, \operatorname{LAST}[a]\right)\right)$. The number of nodes in the first $l$ levels of $\mathscr{T}^{l}$ can be computed from Proposition 3. Cholevel returns the $l$ with the maximum $\Delta(l)$.

\section{Time and space complexities}

Theorem 2 Let $P$ be a set of L-length strings. The level $1 \leqslant l \leqslant L$ such that $\mathscr{T}^{l}$ has the minimum number of states can be computed in $O(\|P\|)$ time and $O(|P| \log |P|)$ space.

Proof: The algorithm Cholevel computes $\mathrm{ML}(P)$ by finding the maximum among $\Delta(1), \ldots, \Delta(L)$. For the running-time analysis, we divide the time into two components. The first component consists of generating $N S$ from $F S$ and $D P$ values of nodes of $N S$ from that of $F S$. It takes time proportional to the number of visited nodes. The total number of visited nodes is just the number of nodes in $\mathscr{T}$, which is $O(\|P\|)$.

The second component consists of building the RMQ structure for $D P$ values of nodes in NS and computing RMQ of each node and its precedent in $N S$. It takes time proportional to the number of visited nodes. The total number of visited nodes is the number of nodes in $\mathscr{T}$. According to Refs. 9-12, RMQ structure is built in linear time and answers the query in $O(1)$ time. The time is $O(\|P\|)$. Thus the total time of the two components is $O(\|P\|)$.

The space used by $F S$ and NS is not greater than $|P|$. The total number of elements in $\operatorname{PatSet}(n)$ of nodes in $F S, N S$, and $D P$ is not greater than $|P|+$ 1. Each entry in these arrays, index or value, uses $O(\log |P|)$ bits. The total used space is $O(|P| \log |P|)$ bits.

\section{EXPERIMENTS}

We implemented the algorithms in $\mathrm{C}$ to compare with previous work. We conducted experiments on a machine with an Intel i7-4610M Haswell $3.0 \mathrm{GHz}$ processor, 8 GB RAM, running 64 bit CentOS 7. Programs are compiled by GCC. We tested with random pattern sets that are generated using the uniform random distribution. The parameters of pattern sets include the number of patterns $|P|$, the length of each pattern $|p|$, and the size of alphabets $|\Sigma|$. 
Table 1 Results of pattern sets of 4000 patterns when each pattern is of length 20 . The size of alphabets ranges from 2-128.

\begin{tabular}{lrrrrrrr}
\hline$|\Sigma|$ & 2 & 4 & 8 & 16 & 32 & 64 & 128 \\
\hline Trie & 36794 & 59346 & 66913 & 70296 & 72320 & 72790 & 75220 \\
Greedy & 30355 & 52976 & 62641 & 68340 & 71176 & 71503 & 74564 \\
& $(18 \%)$ & $(11 \%)$ & $(6 \%)$ & $(3 \%)$ & $(2 \%)$ & $(2 \%)$ & $(0.87 \%)$ \\
O-Trie & 28979 & 52744 & 62397 & 67613 & 71058 & 71503 & 74523 \\
& $(21 \%)$ & $(11 \%)$ & $(7 \%)$ & $(4 \%)$ & $(2 \%)$ & $(2 \%)$ & $(0.93 \%)$ \\
\hline
\end{tabular}

Table 2 Results on pattern sets of 4000 patterns when the size of alphabet is 4 . The length of patterns ranges from 10-140.

\begin{tabular}{lrrrrrrr}
\hline$|p|$ & 10 & 20 & 40 & 60 & 80 & 100 & 140 \\
\hline Trie & 19280 & 59271 & 139477 & 219216 & 299516 & 379305 & 539307 \\
Greedy & 17543 & 53016 & 124365 & 196254 & 267023 & 339631 & 483063 \\
& $(9 \%)$ & $(11 \%)$ & $(11 \%)$ & $(10 \%)$ & $(11 \%)$ & $(10 \%)$ & $(10 \%)$ \\
O-Trie & 17313 & 52821 & 124398 & 195950 & 264484 & 340048 & 482880 \\
& $(10 \%)$ & $(11 \%)$ & $(11 \%)$ & $(11 \%)$ & $(12 \%)$ & $(10 \%)$ & $(10 \%)$ \\
\hline
\end{tabular}

We compared the number of nodes of tires and the O-Tries generated by the greedy heuristic ${ }^{7}$ with our method. The results are given in Table 1Table 5, where each entry is the number of nodes of a trie or O-Trie. The improvement ratio between an O-Trie $T$ and a trie $\mathscr{T}$ is defined as $1-|T| /|\mathscr{T}|$. We give the improvement ratios in the tables under the size of each O-Trie, each ratio is expressed as a percentage. The results for random patterns are given in Table 1-Table 3.

The results for real patterns are given in Table 4 and Table 5. The DNA sequences are from Homo sapiens chromosome 21. The English texts are from the novel 'Mona Lisa Overdrive'. The length of patterns is 40 symbols. The patterns are consecutive strings picked from texts. In all cases (Table 1Table 5), our method generates O-Tries that are smaller than that of the greedy method. Table 1 shows that when the size of the pattern sets is fixed, both methods perform better on short alphabets. For large alphabets, a better level of space saving can be achieved on pattern sets of larger size, which is implied by Table 5 .

\section{Discussion}

The results of Table 1 show the improvement achieved by the new algorithm and the greedy algorithm over the trie. The improvements decline as

Table 3 Results on pattern sets of patterns of length 20 on alphabet of size 16. The number of patterns in each set ranges from 100-12 000 .

\begin{tabular}{lrrrrrrr}
\hline$|P|$ & 100 & 500 & 1000 & 2000 & 4000 & 8000 & 12000 \\
\hline Trie & 1892 & 9204 & 18127 & 35705 & 70275 & 138368 & 205600 \\
Greedy & 1837 & 8925 & 17592 & 34649 & 68320 & 134243 & 199197 \\
& $(3 \%)$ & $(3 \%)$ & $(3 \%)$ & $(3 \%)$ & $(3 \%)$ & $(3 \%)$ & $(3 \%)$ \\
O-Trie & 1825 & 8878 & 17515 & 34463 & 67544 & 132523 & 196695 \\
& $(4 \%)$ & $(4 \%)$ & $(3 \%)$ & $(3 \%)$ & $(4 \%)$ & $(4 \%)$ & $(4 \%)$ \\
\hline
\end{tabular}

Table 4 Results on DNA patterns of length 40.

\begin{tabular}{lrrrrrrr}
\hline$|P|$ & 100 & 500 & 1000 & 2000 & 4000 & 8000 & 12000 \\
\hline Trie & 3750 & 18034 & 35660 & 70316 & 138537 & 273527 & 406533 \\
Greedy & 3394 & 15990 & 31638 & 62568 & 123285 & 243505 & 360908 \\
& $(10 \%)$ & $(11 \%)$ & $(11 \%)$ & $(11 \%)$ & $(11 \%)$ & $(11 \%)$ & $(11 \%)$ \\
O-Trie & 3312 & 15969 & 31594 & 62286 & 123193 & 242922 & 360388 \\
& $(12 \%)$ & $(11 \%)$ & $(11 \%)$ & $(11 \%)$ & $(11 \%)$ & $(11 \%)$ & $(11 \%)$ \\
\hline
\end{tabular}


Table 5 Results on English patterns of length 40.

\begin{tabular}{lrrrrrrr}
\hline$|P|$ & 100 & 500 & 1000 & 2000 & 4000 & 8000 & 12000 \\
\hline Trie & 3889 & 19098 & 37763 & 74594 & 147251 & 290565 & 421917 \\
Greedy & 3772 & 18519 & 36622 & 72207 & 141974 & 279676 & 405949 \\
& $(3 \%)$ & $(3 \%)$ & $(3 \%)$ & $(3 \%)$ & $(4 \%)$ & $(4 \%)$ & $(4 \%)$ \\
O-Trie & 3775 & 18414 & 36496 & 71890 & 141376 & 278344 & 403529 \\
& $(3 \%)$ & $(4 \%)$ & $(3 \%)$ & $(4 \%)$ & $(4 \%)$ & $(4 \%)$ & $(4 \%)$ \\
\hline
\end{tabular}

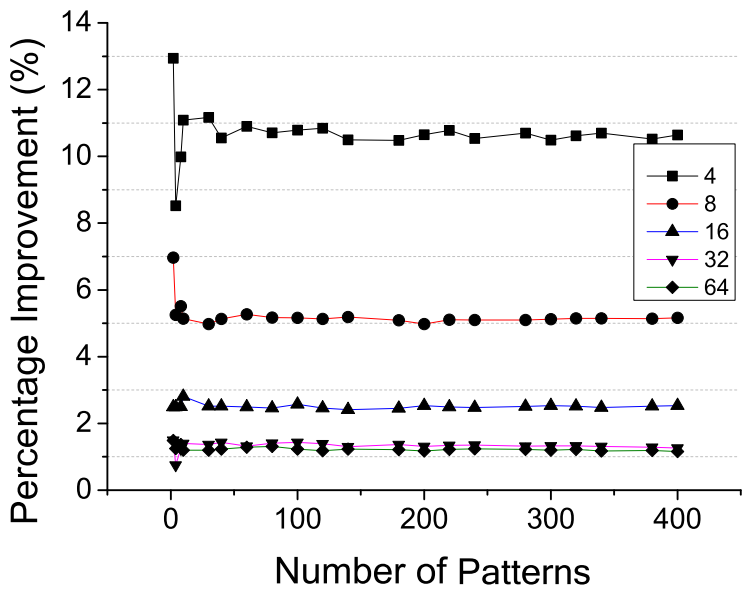

Fig. 4 Results of pattern sets of 2-400 patterns when each pattern is of length 100. The size of alphabets ranges from 4-64.

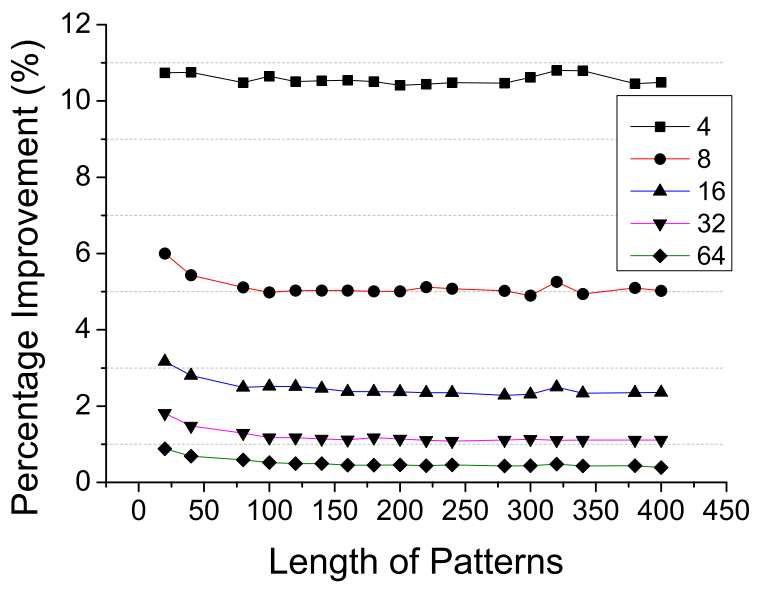

Fig. 5 Results of pattern sets of 200 patterns, the length of patterns ranges from 20-400. The size of alphabets ranges from 4-64.

$|\Sigma|=\sigma$ increases. The results in Table 2-Table 5 show that for a given alphabet the improvement ratios are close to each other, and thus unrelated to the length of patterns or the number of patterns. The ratios of different alphabets are close to ratios

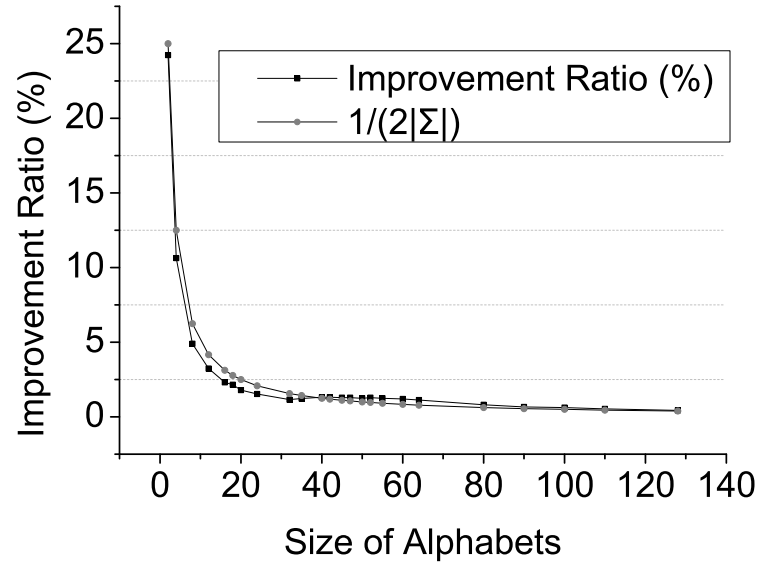

Fig. 6 Results of pattern sets of 200 patterns of length 300 . The size of alphabets ranges from 2-128.

of alphabets shown in Table 1 . To verify the surmise, we conducted more experiments. We give the results in Fig. 4 and Fig. 5 where each data point is the median of improvement ratios of ten trials. The results imply that the size of alphabet determines the improvement ratio, the ratio equals $1 / 2|\Sigma|$ in average.

When we select a level with $s$ different letters of the current PatSet, $s$ nodes will be generated. Denote the average number of different letters in a string of $|P|$ symbols as ac $(|P|)$. In the case of two patterns, the probability of two letters in the same position of the two patterns being the same is $1 / \sigma$. The number of nodes of the trie of $P$ is very close to $\|P\|$, for the average length of the common prefix of the two random strings is $1 / \sigma+1 / \sigma^{2}+$ $\cdots+1 / \sigma^{|p|}$, approximately $1 /(\sigma-1)$. The trie has $2|p|+1-1 /(\sigma-1)$ nodes in average. But the O-Tries for two patterns are smaller, for the average number of positions with the same symbol of two random strings is $|p| / \sigma$. The O-Trie have $2|p|+1-|p| / \sigma$ nodes in average.

We give an upper bound of $\mathrm{ac}(|P|)$ as follows. Partition the letters in a level to a set of disjoint pairs. In average there are $|P| / 2 \sigma$ pairs in which the letters 
are the same. Thus ac $(|P|) \leqslant|P|-|P| / 2 \sigma$. Hence for a node $q$ in an O-Trie, the mean of $A(q)$ is not greater than $|\operatorname{PatSet}(q)|(1-1 / 2 \sigma)$, here $A(q)$ is the number of children of $q$. In average $|A(q)| /|\operatorname{PatSet}(q)| \leqslant 1-$ $1 / 2 \sigma$. Thus the improvement ratio of O-Trie over $\|P\|$ is greater than $1 / 2 \sigma$ with high probability. With the length of patterns grows, $|\operatorname{Trie}(P)| /\|P\|$ is near 1. The average improvement ratio of $\mathrm{O}$-Tries over tries is near $1 / 2 \sigma$ with high probability.

The experiments also show that the improvement ratio is mainly determined by the size of alphabets. We conducted experiments testing a wide range of combinations of $|P|$ and $|p|$. The result in Fig. 6 depicts the ratios of pattern sets on different sizes of alphabets with a fixed $|P|$ and $|p|$ and the curve $y=1 / 2|\Sigma|$. In the experiments, the ratio is close to $1 / 2|\Sigma|$.

\section{CONCLUSIONS}

We have presented a new heuristic for full trie minimization based on rearranging the symbols of strings, which is an efficient method to compute the heuristic function. Further research includes a thorough analysis of the quality of solutions of the method. It is interesting to design pattern matching algorithms using O-Tries. Some orders of O-Tries may help to skip more input symbols. We can use these O-Tries to speed up the dictionary lookup or dictionary matching.

Acknowledgements: This work is supported by the fund of development and reform commission of Jilin province No. 2019C053-10 and the fund of the education Department of Jilin province No. JJKH20190162KJ.

\section{REFERENCES}

1. Fredkin E (1960) Trie memory. Commun ACM 3, 490-9.
2. Morrison DR (1968) PATRICIA - practical algorithm to retrieve information coded in alphanumeric. $J$ ACM 15, 514-34.

3. Aho AV, Corasick MJ (1975) Efficient string matching: An aid to bibliographic search. Commun ACM 18, 333-40.

4. Belazzougui D (2010) Succinct dictionary matching with no slowdown. In: Amir A, Parida L (eds) Combinatorial Pattern Matching, CPM 2010, Lecture Notes in Computer Science, 6129, Springer, Berlin, Heidelberg, pp 88-100.

5. Rotwitt T Jr, deMaine PAD (1971) Storage optimization of tree structured files representing descriptor sets. In: SIGFIDET '71 Proceedings of the 1971 ACM SIGFIDET (SIGMOD), Workshop on Data Description, Access and Control, pp 207-17.

6. Comer D, Sethi R (1977) The complexity of trie index construction. J ACM 24, 428-40.

7. Comer D (1979) Heuristics for trie index minimization. ACM Trans Database Syst 4, 383-95.

8. Comer D (1981) Analysis of a heuristic for trie minimization. ACM Trans Database Syst 6, 513-37.

9. Harel D, Tarjan RE (1984) Fast algorithms for finding nearest common ancestors. SIAM J Comput 13, 338-55.

10. Berkman O, Vishkin U (1993) Recursive star-tree parallel data structure. SIAM J Comput 22, 221-42.

11. Bender MA, Farach-Colton M (2000) The LCA problem revisited. In: Gonnet GH, Viola A (eds) LATIN 2000: Theoretical Informatics, Lecture Notes in Computer Science, 1776, Springer, Berlin, Heidelberg, pp 88-94.

12. Fischer J, Heun V (2006) Theoretical and practical improvements on the rmq-problem, with applications to LCA and LCE. In: Lewenstein M, Valiente G (eds) Combinatorial Pattern Matching, CPM 2006, Lecture Notes in Computer Science, 4009, Springer, Berlin, Heidelberg, pp 36-48. 